\title{
COMO FAZER PESQUISAS CIENTÍFICAS NA ESCOLA? UM GUIA PARA PROFESSORES
}

\section{HOW TO DO SCIENTIFIC RESEARCH IN THE SCHOOL? A GUIDE FOR TEACHERS.}

FERRARI, Carlos Kusano Bucalen ${ }^{1}$

\begin{abstract}
RESUMO
Devido à escassez na formação científica de professores, o presente artigo tem como objetivo revisar brevemente conceitos gerais da pesquisa científica para educadores e professores. Além de discutir a diferença entre ciência e senso comum, ciência básica e aplicada, como classificar um projeto de pesquisa, que fontes de informação científica utilizar e definir o problema de pesquisa, o presente trabalho serve como guia para o desenvolvimento de projetos de pesquisa e trabalhos monográficos acadêmicos em qualquer área do conhecimento (monografias de conclusão de curso, dissertações e teses). É fundamental que a pesquisa científica seja incorporada no cotidiano escolar e na formação continuada de educadores e professores, para que possam ser fomentados os avanços necessários ao conhecimento humano e a derrocada do senso comum e de informações falsas.
\end{abstract}

PalaVRas Chave: metodologia científica; pesquisa básica; pesquisa aplicada; informação; projeto de pesquisa.

\section{ABSTRACT}

Due to the scarcity of scientific formation for teachers, this article attempts to briefly review general concepts of scientific research for educators and teachers. Besides discussing the difference between science and common sense, basic and applied science, how to classify a research project, what sources of scientific information to use, and defining the research problem, this paper serves as a guide for the development of research projects in any area of knowledge (undergraduate monographs, master and $\mathrm{PhD}$ theses). It is essential that scientific research be incorporated into daily school life and in the continuing education of educators and teachers, so that the necessary advances in human knowledge and the collapse of common sense and false information can be encouraged.

KEYWORDS: scientific methodology, basic research, applied research, information, research project

\footnotetext{
${ }^{1}$ Universidade Federal do Mato Grosso (UFMT) / Instituto de Ciências Biológicas e da Saúde (ICBS). Araguaia, MT, Brasil. ORCID: https://orcid.org/0000-0001-9325-1260 e-mail: ferrariphd@yahoo.com.br
} 
DOI: $10.12957 /$ e-mosaicos.2020.45084

\section{INTRODUÇÃO}

Embora seja inegável o enorme avanço no sistema de pós-graduação brasileira das últimas 4 décadas, iniciativas para o fomento efetivo e disseminado de uma cultura científica nas escolas, especialmente em regiões mais distantes dos grandes centros urbanos ainda é escassa ou tímida.

Um estudo ilustra o que está acima afirmado e certamente configura uma verdade, mesmo que diversa, em grande parte das escolas de todo o país. Segundo Pires e Prado (2018), a maioria dos professores de uma escola pública, localizada em São Mateus (ES), não teve formação adequada para ensinar ciências e precisa de educação continuada para aprender conteúdos científicos.

Em virtude da ampla disseminação global de informações falsas, fraudulentas e mal-intencionadas, sem respaldo científico, como o risco de adquirir autismo e outras doenças decorrentes da vacinação (VASCONCELLOS-SILVA et al., 2015) ou que o planeta Terra é plano (CAMARGO-JR, 2018), é evidente que a popularização e a comunicação da ciência têm sido tímidas e ineficazes.

A disseminação de notícias cientificamente falsas, assim como o corte de recursos financeiros de ciência, tecnologia e educação demonstram que falta ainda muito diálogo com a sociedade para mostrar o valor da ciência (MASSARANI; ARARIPE, 2019). Porém, como enfatizam as mesmas autoras, na presença de recursos financeiros, os cientistas brasileiros foram muito ágeis em pesquisar e descobrir as possíveis relações do vírus Zika com a microcefalia, mostrando que a ciência tem um valor inestimável para a prevenção das doenças e bem-estar da população.

Camargo-Jr (2018) sugeriu que a cultura de se preocupar apenas em publicar artigos (publish or perish), precisa ser revista, sendo urgente que os cientistas promovam o alfabetismo científico da população.

Deste modo, é fundamental ensinar e disseminar os diversos tipos de ciência para distingui-las do senso comum, fomentando atividades científicas de pesquisa desde a mais tenra idade, especialmente nos ambientes escolares.

Assim sendo, o presente artigo teve como objetivo elaborar um guia teóricometodológico e prático para que professores possam desenvolver seus projetos com seus alunos desde os últimos anos do Ensino Fundamental.

Para que este guia seja de fácil utilização, evitaram-se os jargões científicos, bem como uma abordagem exaustiva de métodos e técnicas de pesquisa.

\section{Fundamentos Teóricos e Metodológicos da Investigação Científica}

\section{CuIdAdO COM O SENSO COMUM}

O senso comum tem tido ênfase na atualidade devido aos movimentos antivacinação e terraplanistas que se baseiam em fatos inverídicos (fake news), ideias e percepções errôneas sobre os fenômenos da vida. Quando uma pessoa, baseada em 
observações simplistas em sua rua ou bairro, diz que nascem mais meninas, isto pode ser apenas um senso comum. É necessário verificar as estatísticas de nascimento para fazer tal afirmação. Porém, hoje ainda nascem mais meninos que meninas, mas o sexo masculino apresenta maior mortalidade e menor esperança de vida ao nascer, o que explica maior quantidade de viúvas que viúvos. No Brasil, segundo o Instituto Brasileiro de Geografia e Estatística (IBGE), a razão de sexos (sex ratio), também conhecida como coeficiente de masculinidade, é de 105 meninos nascidos vivos para cada 100 meninas nascidas vivas. Em 2017, por exemplo, segundo as estatísticas do registro civil, compiladas e analisadas pelo IBGE, nasceram 1.518.444 meninos e 1.444.005 meninas (IBGE-SIDRA, 2019a). Este resultado está de acordo com o estudo clássico de Hawley (1959), sendo encontrado na maioria dos países do mundo, incluindo os do Norte da Europa e Japão (FELLMAN, 2015; FUKUDA et al., 2014). Em outros países, como Argentina, Chile, Austrália, Finlândia, Reino Unido, Alemanha, Rússia, Ásia Central, Egito e outros, a razão de sexos está em declínio ou vem aumentando, mas continuam nascendo mais meninos que meninas (Grech, 2014; Grech, 2015). Como a mortalidade é maior no sexo masculino, especialmente na faixa etária dos 18 a 24 anos, há um equilíbrio na razão dos sexos dos 25 aos 49 anos, mas a população feminina predomina na velhice (IBGE-SIDRA, 2019b; MELO et al., 2017).

Aprofundando as características do senso comum, é importante ressaltar que este tipo de conhecimento é uma versão simplista, passiva, fragmentada, mecânica, desarticulada, implícita e incoerente da verdade (SAVIANI, 1980).

\section{UTILIZANDO A ÁRVORE DO CONHECIMENTO DO CNPQ}

Inicialmente, o professor deve escolher uma temática junto aos seus alunos e classificá-la conforme a árvore do conhecimento do Conselho Nacional de Desenvolvimento Científico e Tecnológico (CNPQ: http://lattes.cnpq.br/web/dgp/arvore-do-conhecimento). A seguir são apresentados dois exemplos de temas e como eles podem ser classificados segundo o CNPQ.

Um professor resolve propor aos alunos tentar desenvolver um método químico ou físico para filtrar ou remover os agrotóxicos da água de abastecimento público e residencial. Neste caso, o projeto pode ter como área do conhecimento principal do CNPQ, as "Ciências exatas e da terra", subárea "Química analítica" e seção "Análise de traços e química ambiental". Porém, o mesmo tema poderia ser também classificado como sendo da grande Área "Engenharias", subárea "Engenharia Sanitária" e seção "Tratamento de águas de abastecimento e Residuárias". Esta temática ainda poderia ser classificada como sendo da grande área de "Ciências da Saúde", subárea "Farmácia" e seção "Análise toxicológica". Este exemplo, feito de modo proposital, mostra como um mesmo tema pode estar presente e ser classificado em diferentes áreas afins.

Mas, outro exemplo pode ser útil também. Consideremos que uma professora pretenda estudar como textos literários (poemas, contos, romances) podem ajudar os alunos a compreender melhor os animais, ou seja, o estudo da Zoologia. Neste caso, embora sua temática tenha relação com o ensino de Zoologia, a classificação mais 
DOI: $10.12957 / \mathrm{e}-\mathrm{mosaicos} .2020 .45084$

adequada seria grande área "Ciências Humanas", subárea "Educação" e seção "Ensinoaprendizagem" e subseção "Métodos e técnicas de ensino".

\section{DEFININDO O PROBLEMA DE PESQUISA}

Embora a professora ou o professor tenham um tema e um problema prédefinidos, é importante estimular os alunos a raciocinar sobre o problema de pesquisa. Isto é importante não apenas para estimular a curiosidade, mas inclusive desenvolver desde cedo a reflexão, a iniciativa e o protagonismo dos estudantes.

Mesmo que não seja obrigatório definir um tema inédito, devido à enorme variedade de temas e problemas que necessitam de uma busca por soluções, é fundamental que o professor esteja aberto e aceite o desafio de tentar desenvolver com seus alunos um tema e/ou problema que seja original e inédito.

Um tema pode ser "trânsito seguro", e possíveis problemas de pesquisa podem ser fatores que aumentam a probabilidade (fatores de risco) de acidentes de trânsito no município e, uma vez conhecendo tais fatores, quais são as medidas concretas para tentar reduzir acidentes, lesões e mortes no trânsito do seu município? As medidas concretas, como, por exemplo, estudar um tipo de material, para uso como asfalto, que possa dar maior aderência aos veículos e evitar acidentes, pode ser um problema de pesquisa. No mesmo exemplo dos acidentes de trânsito, um problema de pesquisa pode ser como desenvolver um aplicativo para smartphone ou notebook e tablets que avisa os sistemas de emergência (bombeiros, SAMU, polícia, etc.) sobre a ocorrência de um acidente, o local, os afetados e quais possíveis gravidades sofridas pelos envolvidos, de modo que o socorro venha melhor preparado para o atendimento.

Um outro problema pode ser como aprender a cultivar e consumir seu próprio alimento. A epidemia de obesidade cujo enfrentamento é muito escasso, precisa ser combatida pelo cultivo de hábitos alimentares saudáveis (FERRARI, 2018) e, para isso, é necessário conhecer melhor os alimentos, como eles são produzidos e aprender a preparar refeições saudáveis. Uma das explicações para a rápida epidemia de obesidade no Brasil é que a população optou por alimentos ultraprocessados (ricos em gordura, açúcar e sal), que já se encontram prontos para o consumo, em detrimento de escolher, higienizar e preparar sua própria comida. Reduzir o consumo de alimentos ultraprocessados e aumentar a ingestão de alimentos in natura ou de baixo processamento são preconizados pelo Guia Alimentar da População Brasileira (BRASIL, 2014). Os alimentos ultraprocessados aumentam o risco de obesidade e doenças crônicas (doenças cardiovasculares, circulatórias e diabetes) (LOUZADA et al., 2015). $O$ professor pode estimular os alunos a pesquisar quais alimentos mais nutritivos podem ser cultivados na horta da escola e quais são as facilidades e dificuldades em se cultivar certas espécies comestíveis. A horta escolar, além de exemplo de sustentabilidade, cumpre com pelo menos quatro dos 17 objetivos do desenvolvimento sustentável (ODS), que são fome zero e agricultura sustentável, educação de qualidade, saúde e bem-estar (a importância da alimentação para a saúde) e cidades e comunidades sustentáveis, além de induzir, pelo menos indiretamente, outro ODS, a erradicação da pobreza (ONU/BRASIL, 2015). 
DOI: $10.12957 / \mathrm{e}-\mathrm{mosaicos} .2020 .45084$

De toda forma, é muito importante que o professor abandone as "amarras" acadêmicas e busque junto aos seus alunos estudar problemas reais e que possam ter impacto significativo na vida da população.

\section{Pesquisa básica e pesquisa aplicada}

A pesquisa científica pode ser básica, quando busca aumentar o conhecimento sobre o mundo humano e a natureza, ou aplicada, sendo aquela que visa também produzir novos conhecimentos, mas que tenham aplicação prática para solucionar problemas específicos. A pesquisa básica é fundamental para que seja possível fazer pesquisa aplicada. Por exemplo, uma pesquisa básica visa descobrir as qualidades físicas (temperatura, dilatação, pressão, etc.) de diversos tipos de materiais que poderão ser estudados, posteriormente, para a fabricação de cadeiras, móveis, roupas, smartphones, etc. (pesquisa aplicada).

A pesquisa básica e a pesquisa aplicada também têm sido denominadas de não dirigida e dirigida, respectivamente (LOPES, 1991; BOLZANI, 2016).

Milhares de pessoas, que desconhecem a ciência e baseiam-se em sensos comuns, acreditam que pesquisa básica é inútil ou de pouco valor, enquanto que se deveria investir apenas em pesquisas aplicadas.

Um estudo recente, desenvolvido por pesquisadores da Universidade Federal de Goiás, investigando a cera do ouvido humano identificou 158 compostos orgânicos, sendo 27 marcadores ou indicadores específicos do câncer (BARBOSA et al., 2019). Este estudo científico seria básico ou aplicado? Esta pesquisa abre as portas para um diagnóstico não-invasivo do câncer e representa um exemplo de que pesquisa básica e aplicada são, muitas vezes, inseparáveis.

\section{Pesquisa quantitativa e Qualitativa}

Um projeto pode planejar o desenvolvimento de pesquisa quantitativa, cujo objetivo é quantificar ou estimar numericamente um problema de pesquisa para sistematizá-lo e analisá-lo (GIL, 1999). Por exemplo, um professor e seus estudantes visam desenvolver um dispositivo elétrico que possa atrair insetos e destruí-los, visando matar mosquitos transmissores de doenças, como a dengue e a malária. Para fazer isso, é necessário quantificar o número de insetos mortos.

Porém, a pesquisa também pode ser qualitativa, quando busca-se saber quais são as características, qualidades e expectativas quanto a determinado fenômeno. Neste tipo de pesquisa, geralmente são analisados depoimentos ou discursos dados pelas pessoas. Entrevistar os alunos para saber a opinião deles de como as aulas da escola poderiam ser mais interessantes, colhendo depoimentos, é um exemplo de pesquisa qualitativa (GIL, 1999). 
DOI: $10.12957 /$ e-mosaicos.2020.45084

É importante ressaltar que em inúmeras ocasiões utilizam-se abordagens mistas, ou seja, quali-quantitativas para se estudar fenômenos que envolvem populações humanas.

\section{Escolhendo o TiPo de Pesquisa}

Duas abordagens teóricas são contempladas na pesquisa bibliográfica e de dados, que utiliza material já publicado (atlas, livros, artigos, sistemas de dados do IBGE e do Ministério da Saúde, etc.), assim como na pesquisa documental. Esta última utiliza materiais que ainda não sofreram tratamento analítico, ou seja, não foram discutidos, sistematizados ou publicados em veículos científicos (livros ou revistas científicas). Uma professora e seus alunos podem buscar documentos para tentar construir a Historiografia da cidade ou de uma etnia específica naquele município. Outros detalhes da pesquisa bibliográfica e documental podem ser encontrados em Gil (2017).

A tabela 1 sumariza fontes de informação para pesquisa bibliográfica e pesquisa documental e a tabela 2 traz as bases indexadoras e revistas científicas.

Tabela 1. Fontes de informação para investigação científica

\begin{tabular}{|c|c|}
\hline Tipo de informação & Descrição e características \\
\hline \multirow[t]{4}{*}{ Demografia } & $\begin{array}{l}\text { Censo do IBGE (https://ibge.gov.br/ } \\
\text { https://cidades.ibge.gov.br/): }\end{array}$ \\
\hline & $\begin{array}{l}\text { município, gênero, idade, etnia, ocupação, renda e } \\
\text { escolaridade }\end{array}$ \\
\hline & Nascimentos: por região, incluindo peso ao nascer \\
\hline & Óbitos: por região, idade, causa de morte, local, etc \\
\hline \multirow[t]{2}{*}{ Estatísticas vitais } & Taxas de mortalidade (perinatal, infantil, etc) \\
\hline & Taxas de nascimento e fertilidade \\
\hline \multirow[t]{2}{*}{ Morbidade } & Doenças de notificação compulsória \\
\hline & Registros de câncer e hospitalares \\
\hline \multirow{6}{*}{$\begin{array}{l}\text { Dados sociais e } \\
\text { ambientais }\end{array}$} & Estatísticas de acidentes de trânsito \\
\hline & $\begin{array}{l}\text { Estatísticas sobre a violência (Atlas da violência do } \\
\text { IPEA). }\end{array}$ \\
\hline & Poluição do ar \\
\hline & Água potável \\
\hline & Ruídos \\
\hline & $\begin{array}{l}\text { Dados sobre espécies domiciliadas: baratas, } \\
\text { mosquitos, ratos, etc. }\end{array}$ \\
\hline
\end{tabular}


DOI: $10.12957 /$ e-mosaicos.2020.45084

Patentes

Publicações

governamentais

Agências de pesquisa

Histórica

Bases indexadoras e portais de periódicos científicos
Instituto Nacional de Propriedade Intelectual (INPI, Brasil), United States Patent and Trademark OfficeUSPTO (Estados Unidos, https://www.uspto.gov/), European Patent Registry-EPR (Europa, https://www.epo.org/searching-forpatents/legal/register.html), World Intelectual Property Organization (PatentScope: https://patentscope.wipo.int/search/pt/search.jsf ), etc

Diversos tipos de informação sobre políticas públicas nas mais diferentes áreas (educação, esporte, saúde, cidadania, justiça, meio ambiente, cultura da paz, etc.)

Diversos

Arquivos públicos e privados, museus, centros culturais, teatros, igrejas, etc.

Vide tabela 2

Tabela 2. Base de dados para informação científica e portais de revistas científicas

\begin{tabular}{ll} 
Base de dados & Área/Endereço \\
\hline arXiv & Ciências exatas \\
& https://arxiv.org/
\end{tabular}

Base de dados BDPA: https://www.bdpa.cnptia.embrapa.br/consulta/ da Pesquisa Agropecuária

Biblioteca

Saúde: https://bvsalud.org/ virtual em saúde (BVS)

CAB Abstracts Ciências da vida e da natureza, ciências agrárias, ciência de alimentos, veterinária, medicina, biomédicas, etc

https://www.cabi.org/publishing-products/online-informationresources/cab-abstracts/

Cochrane $\quad$ Evidências científicas biomédicas e de saúde:

https://www.cochrane.org/ 
DOI: $10.12957 /$ e-mosaicos.2020.45084

Domínio público http://www.dominiopublico.gov.br/pesquisa/PesquisaObraForm.d $\underline{0}$

Diretório de Livros: https://www.doabooks.org/

livros de acesso

livre (DOAB)

EDUBASE Educação, biblioteconomia, ciência da informação e afins http://portal.edubase.modalbox.com.br/index.php/site/sobre/

Food Science Ciência e Tecnologia de Alimentos; https://www.ifis.org/fsta and Technology Abstracts (FSTA)

Literatura Latinoamerican Saúde: http://lilacs.bvsalud.org/ a em Ciências da Saúde (LILACS)

Localizador de

Saúde: https://bvsalud.org/portal-lis/ Informação em Saúde (LIS)

Medline Saúde, biológicas e biomédicas;

(Biblioteca Nacional de Medicina dos Estados Unidos)

Periódicos https://www.ncbi.nlm.nih.gov/pubmed/

é possível também pesquisar em português via BVS

Capes

SportDiscus

Todas as áreas

http://www.periodicos.capes.gov.br/

Ciências do Esporte https://www.ebsco.com/products/researchdatabases/sportdiscus

Portais de revistas

Diretório de revistas de

DOAJ: https://doaj.org/ acesso livre. (Directory of open acess journals) Latindex Periódicos latino-americanos de todas as áreas https://www.latindex.org/latindex/inicio 
DOI: $10.12957 /$ e-mosaicos.2020.45084

Pubmedcentral Saúde, biológicas e biomédicas:

https://www.ncbi.nlm.nih.gov/pmc/

Redalyc Revistas latino-americanas de todas as áreas

http://www.redalyc.org/home.oa

Scielo

Todas as áreas: $\underline{\text { www.scielo.org ; www.scielo.br }}$

Porém, professores de ciências podem desenvolver pesquisas experimentais, envolvendo definição de um objeto de estudo, o problema de estudo (da pesquisa), a seleção de variáveis que podem influenciar o objeto de estudo (idade, sexo, temperatura, umidade, pressão, etc.) e a definição de formas de controle e de observação dos efeitos que as variáveis produzem no objeto. Por exemplo, uma professora pode estar interessada, junto aos seus alunos, em estudar o uso de restos de um alimento que possa ser utilizado para a produção de uma sacola que seja totalmente biodegradável e possa substituir sacolas plásticas. Este grupo terá que estudar fatores como resistência do material, durabilidade do produto e tempo de degradação do material, dentre outras variáveis físicas e químicas. Dando outro exemplo, o professor de Educação Física pode quantificar o peso, a estatura e o índice de massa corporal para avaliar qual é a porcentagem de alunos que tem peso normal, sobrepeso ou obesidade. Isto é importante para que os professores de Educação Física, bem como os de Ciências e Biologia possam ensinar sobre a importância de ter hábitos alimentares saudáveis e prática de atividades físicas para promover a saúde e reduzir o excesso de peso.

\section{REVISÃO CRÍTICA DA LITERATURA CIENTÍFICA OU INVESTIGAÇÃO DOCUMENTAL?}

Professores da área de humanidades e ciências sociais aplicadas podem desenvolver seus projetos levando os alunos a fazer uma leitura crítica e uma síntese de literatura científica ou investigação de documentos. É claro que os alunos ainda leem pouco e, portanto, serão iniciados na leitura e produção textual, orientadas pelo docente.

Nas outras áreas do conhecimento, também é importante realizar uma revisão bibliográfica para dar embasamento ao projeto e mesmo certificar-se que o mesmo é original e inédito.

Constituem critérios importantes de aceitabilidade do trabalho acadêmico de revisão a confiabilidade nos dados colhidos (veja as fontes nas Tabelas 1 e 2), a análise crítica dos resultados com vistas à formulação de um consenso e uma escrita sem erros ortográficos e sintáticos. 
DOI: $10.12957 /$ e-mosaicos.2020.45084

\section{PesQuisa eXPERIMENTAL}

Neste tipo de pesquisa, para atingir os objetivos, são necessários realizar diversos tipos de experimentos ou ensaios experimentais em que são medidas ou mensuradas variáveis pré-definidas em razão de uma intervenção ou indução, sendo um tipo de pesquisa quantitativa. Ex.: no caso de um dispositivo eletrônico antimosquitos, os professores e seus alunos vão experimentar quais intensidades elétricas são mais eficazes na indução da morte dos insetos e qual voltagem é melhor (110 ou 220v), além de outros fatores físicos.

\section{Pesquisa de campo}

A pesquisa de campo é aquela em que os pesquisadores vão ao local de pesquisa, coletam e registram materiais e espécimes (vegetais, minerais, fósseis) e realizam medidas do ambiente (temperatura, umidade, densidade do solo, pH, etc). Uma pesquisa de intenções de voto nas eleições também é uma investigação de campo, assim como uma pesquisa para verificar o perfil de saúde dos usuários de uma unidade básica de saúde do Sistema Único de Saúde (SUS). Para um aprofundamento sobre a pesquisa de campo e pesquisa quantitativa considere a leitura de Piana (2009).

\section{ESCREVENDO O PROJETO DE PESQUISA}

Algumas décadas atrás, esperava-se que os autores fizessem um projeto de pesquisa longo com 10 ou mais páginas e pelo menos 20 referências bibliográficas. concisos.

Hoje em dia, sabe-se da importância de projetos densos, completos, mas

Um projeto deve conter as seguintes partes (SILVA et al., 2004):

1) Título;

2) Introdução;

3) Definição do problema;

4) Objetivos (geral e específicos);

5) Justificativa ou importância da pesquisa;

6) Delimitação do estudo;

7) Metodologia;

8) Referencial teórico ou revisão de literatura;

9) Referências;

10) Cronograma de trabalho. 
DOI: $10.12957 / \mathrm{e}-\mathrm{mosaicos} .2020 .45084$

\section{QUAIS SÃo AS ETAPAS DA PESQUISA CIENTÍFICA?}

\section{INTRODUÇÃO E DESENVOLVIMENTO DO PROBLEMA DE PESQUISA}

A pesquisa científica inicia-se com a Introdução. No caso do projeto escolar, pode ser, em média, de duas a três páginas em que os autores vão descrever o tema, já trazendo pelo menos duas referências bibliográficas recentes e pertinentes. Na introdução os autores expõem o tema e o problema a serem desenvolvidos.

No desenvolvimento do problema, os autores formulam uma pergunta ou questão da pesquisa e elaboram uma resposta provisória, conhecida como hipótese. Assim, o desenvolvimento do trabalho científico testará a validade da hipótese e seus resultados poderão confirmá-la ou rejeitá-la (FERRARI, 1982).

A hipótese pode estar implícita ou explícita no trabalho. É importante ressaltar que, nos livros mais antigos e ainda hoje em certas publicações, enfatiza-se que somente pesquisas experimentais ou de levantamento de dados podem ter hipóteses. Na verdade, as pesquisas em humanidades também podem e devem ter hipóteses, visto que a confirmação das mesmas é essencial para a construção das Teorias em qualquer área do conhecimento (BARROS, 2008).

\section{Objetivos}

O objetivo geral tem como função testar ou verificar a validade da hipótese.

O objetivo deve ser claro e conciso. O professor e seus alunos precisam saber muito bem qual é o objetivo. De modo geral, a principal meta a ser atingida pelo pesquisador constitui seu objetivo. Deve haver um objetivo geral, podendo existir dois ou três objetivos específicos.

\section{METOdOLOGIA}

Nesta etapa, os métodos de pesquisa e os procedimentos operacionais (técnicas) a serem desenvolvimentos tem como finalidade cumprir com os objetivos do trabalho (GIL, 2017).

Em estudos experimentais da biologia, física, química, engenharias e outras áreas os autores preferem denominar esta etapa de "Materiais e métodos". Porém, em estudos envolvendo seres humanos não se deve denominar de materiais, mas de pessoas ou sujeitos. Por esta causa, prefere-se chamar esta fase de "Metodologia" que é o conjunto de métodos (conceituais teóricos) e técnicas (procedimentos operacionais) a serem desenvolvidas na pesquisa para atingir os objetivos.

É importante descrever de modo detalhado os métodos e técnicas utilizados na coleta de dados, informações, espécies vegetais, documentos, etc.

Os professores devem descrever o local do estudo, o período do estudo, os métodos utilizados (químicos, físicos, biológicos, matemáticos, estatísticos, 
DOI: $10.12957 /$ e-mosaicos.2020.45084

geográficos, históricos, etc.). No caso de pesquisas experimentais, os procedimentos precisam ser detalhados. Ex.: como foi colhida a amostra, se foi de urina, sangue, água ou solo, qual instrumento utilizado para coleta, qual a quantidade de material coletado (em ml, gramas, etc.) e toda a descrição de como foi processado o material para estudo. Exemplos da área de alimentos, biológica, biomédica, educação física, enfermagem, farmacêutica e de saúde podem ser encontrados em diversos estudos (ALVES ET AL., 2015; FRANÇA et al., 2009; FERRARI et al., 2012; FERRARI et al., 2013; SPANHOL; FERRARI, 2016; FERRARI et al., 2017a,b; LAZZAROTTI FILHO et al., 2018; REZENDE et al., 2019; PEREIRA et al., 2019). Para as demais áreas deve-se seguir a descrição metodológica das pesquisas que serviram de embasamento ao projeto.

Na metodologia, também precisa ser descrita, quando for o caso, a análise estatística ou tratamento estatístico a ser realizado com os dados levantados no estudo. Para a análise estatística, além de estimadas a média e o desvio-padrão, também é necessário definir o nível de significância que, em geral, é estabelecido como sendo $p<0,01$ ou $p<0,05$. Há várias décadas, utilizam-se programas estatísticos que estimam o valor de $\mathrm{p}$.

Além dos testes estatísticos, a pesquisa que utiliza seres vivos também precisa de aprovação de comitê de ética em pesquisa. Se utilizar animais de laboratório, necessita de aprovação em comitê de ética em pesquisa animal e, se for com humanos, no respectivo comitê de ética em pesquisa com seres humanos.

Para projetos com seres humanos, é fundamental consultar a comissão nacional de ética em pesquisa (CONEP) do Conselho Nacional de Saúde, vinculado ao Ministério da Saúde (BRASIL, 2019). Além da leitura dos documentos desta página é interessante tirar dúvidas com pesquisadores já familiarizados ou entrar em contato com um comitê de ética da sua região. O projeto deverá ser submetido à Plataforma Brasil e somente poderá ser iniciado após sua aprovação (BRASIL, 2019).

\section{REFERENCIAL TEÓRICO OU REVISÃo DE LITERATURA CIENTÍFICA}

Deve ser focada, densa e completa, porém concisa, trazendo estudos atuais, especialmente dos últimos três ou cinco anos. Não há necessariamente um número de páginas considerado ideal, mas não deve ser longa (mais de 5 páginas, nem muito breve (apenas uma página).

\section{Cronograma e Orçamento}

O projeto deve conter cronograma detalhado das atividades, que pode ser apresentado em apenas uma página.

Considerando-se que professores podem tentar conseguir recursos financeiros para executar seus projetos, é importante descrever no projeto um orçamento contendo material de consumo (papéis, pastas, toner, reagentes químicos, despesas com transporte, etc.), material permanente (equipamentos e instrumentos) e bolsas. 
DOI: $10.12957 /$ e-mosaicos.2020.45084

É importante também ter um orçamento de contrapartida que é o custo de a ser bancado pelo professor e/ou sua escola (impressora, microcomputador, notebook, energia elétrica, água, salário do professor equivalente pela dedicação em horas semanais ao projeto, equipamentos da escola e/ou do professor, etc.). Assim, o orçamento total será o orçamento solicitado somado ao orçamento de contrapartida.

\section{Perspectivas e conclusões}

Embora a escola, especialmente pública, não venha sendo um espaço favorável à docência e ao desenvolvimento de seus professores (PENIN, 1994; FRANCHI, 1995), segundo Nóvoa (1992) e Pereira (2002) é necessário criar ambientes escolares para a formação de professores que exerçam reflexão, investigação científica e promovam transformação social e seu próprio protagonismo. Nesta perspectiva, a pesquisa científica na escola é uma das formas mais eficazes de enfrentamento do sensocomum segundo Perrenoud (1993).

Neste sentido, é urgente e necessário que a Universidade abra seus muros para a comunidade externa, especialmente as escolas, e contribua mais efetivamente para a formação científica dos professores, oferecendo cursos dos mais variados tipos, incluindo estratégias de ensino à distância.

Em 2006, a Comissão Europeia definiu e implementou as oito competências para o aprendizado dos cidadãos europeus, incluindo "aprender a aprender" e "competências básicas em ciência e tecnologia" (COMISSÃO EUROPEIA, 2006). E nós? Até quando continuaremos atrasados?

\section{HOMENAGEM}

Este artigo é uma homenagem póstuma a Levi Bucalem Ferrari, meu pai, Gestor Público Municipal e Estadual eficiente, Sociólogo, Cientista político, ex-diretor de Escola, professor universitário, ex-presidente da União Brasileira de Escritores (UBE) e escritor de livros publicados de poemas, contos e romances. Autor de diversos romances selecionados e premiados com o "O sequestro do senhor empresário" (prêmio APCA), "O inimigo" e "Seduções".

\section{REFERÊNCIAS}

ALVES, J.D.; ROCHA, I.C.; SILVA, L.F.P.; FERRARI, C.K.B. Higienização das mãos: olhar dos profissionais de enfermagem em uma unidade de terapia intensiva do adulto. Revista Uruguaia de Enfermería, v.2, n.10, 12-23, 2015.

BARBOSA, J.M.G.; PEREIRA, N.Z.; DAVID, L.C.; OLIVEIRA, C.G. DE; SOARES, M.F.G.; AVELINO, M.A.G.; OLIVEIRA, A.E. DE; SHOKRY, E.; ANTONIOSI FILHO, N.R. Cerumenogram: a new frontier in cancer diagnosis in humans. Scientific Reports, 9, article number:11722, 2019. Disponível em: https://www.nature.com/articles/s41598-01948121-4 [03/09/2019]. 
DOI: $10.12957 / \mathrm{e}-\mathrm{mosaicos} .2020 .45084$

BARROS, J.D'A. A elaboração textual de hipóteses - uma contribuição ao seu esclarecimento no ensino de metodologia. Revista Educação em Questão, v.33, n.19, 305-328, 2008.

BOLZANI, V.S. Pesquisa básica ou pesquisa aplicada, interligadas em permanente movimento. Qual a melhor para o país? J Ciências 2016;5510:22. Disponível em: http://www.jornaldaciencia.org.br/edicoes/?url=http://jcnoticias.jornaldaciencia.org. br/22-pesquisa-basica-ou-pesquisa-aplicada-interligadas-em-permanente-movimentoqual-a-melhor-para-o-pais/ [30/08/2019].

BRASIL. Ministério da Saúde. Guia alimentar para a população brasileira. Brasília, DF, 2a Ed., 2014.

BRASIL. Ministério da Saúde. Comissão Nacional de Ética em Pesquisa (CONEP). Brasília, 2019. Disponível em: http://conselho.saude.gov.br/plataforma-brasilconep?view=default e em: http://plataformabrasil.saude.gov.br/login.jsf [31/08/2019].

CAMARGO-JR, K.R. DE. Para defender a ciência, é necessário torná-la acessível, inteligível e significativa. Physis Revista de Saúde Coletiva, v.28, n.2, e280201, 2018.

COMISSÃO EUROPEIA. Recomendação 2006/962/CE. In: Eurolex. Disponível em: http://eur-lex.europa.eu/legal-content/PT/TXT/?uri=LEGISSUM\%3Ac11090 [01/09/2019].

FERRARI, A.T. Metodologia da pesquisa científica. São Paulo, McGraw-Hill do Brasil, 1982.

FERRARI, C.K.B.; SAVAZZI, K.; HONORIO-FRANÇA, A.C; FERRARI, G.S.L.; FRANÇA, E.L. Conhecimentos sobre hepatites virais numa amostra de estudantes brasileiros do Vale do Araguaia, Amazônia Legal. Acta Gastroenterologica Latinoamericana, v.42, n.2, 120-126, 2012.

FERRARI, C.K.B.; BRITO, L.F.; OLIVEIRA, C.C.; MORAES, E.V. DE; TOLEDO, O.R. DE; DAVID, F.L. Falhas na prescrição e dispensação de medicamentos psicotrópicos: Um problema de Saúde Pública. Revista de Ciências Farmacêuticas Básica e Aplicada, v.34, n.1, 109-116, 2013.

FERRARI, C.K.B.; PERCÁRIO, S.; SILVA, J.C.C.B.; TORRES, E.A.F.S. An apple plus a Brazil nut a day keep the doctors away: antioxidant capacity of foods and their health benefits. Current Pharmaceutical Design, v.22, 189-195, 2016.

FERRARI, C.K.B.; CARNEIRO, S.S.; ROCHA, E.M. DA; SANTOS, A.L.V. dos. Sedentarismo, estilo de vida e saúde em adolescentes de um município da Amazônia Legal. Revista Inspirar Movimento \& Saúde, v.14, n.3, 28-33, 2017. 
DOI: $10.12957 / \mathrm{e}-\mathrm{mosaicos} .2020 .45084$

FERRARI, C.K.B.; FRANÇA, E.L.; MONTEIRO, L.A.; SANTOS, B.L.; PEREIRA-JUNIOR, A.; HONORIO-FRANÇA, A.C. Chronopharmacological effects of growth hormone on the executive function and oxidative stress response in rats. Iranian Journal of Basic and Medical Sciences, v.20, n.1, 17-22, 2017.

FERRARI, C.K.B. Implementation of public health policies for healthy lifestyles promotion: what Brazil should tell us? Health Promotion Perspectives, v.8, n.3, 243248, 2018.

FRANÇA, E.L.; FELICIANO, N.D.; SILVA, K.A.; FERRARI, C.K.B.; HONORIO-FRANÇA, A.C. Modulatory role of melatonin on superoxide release by spleen macrophages isolated from alloxan-induced diabetic rats. Bratislava Medical Journal, v.110, n.9, 517-522, 2009.

FRANCHI, E.P. (org.). A causa dos professores. Campinas: Ed. Papirus, 1995.

FELLMAN, J. Temporal and regional trends in secondary sex ratio: The Nordic experience. Early Human Development, v.91, n.12, p.801-806, 2015.

FUKUDA, M.; FUKUDA, K.; SHIMIZU, T.; NOBUNAGA, M.; MAMSEN, L.S.; ANDERSEN, C.Y. Climate change is associated with male:female ratios of fetal deaths and newborn infants in Japan. Fertility and Sterility, v.102, n.5, p.1364-1370, 2014.

GIL, A.C. Métodos e técnicas de pesquisa social. São Paulo, 5a ed., Atlas, 1999.

GIL, A.C. Como elaborar projetos de pesquisa. São Paulo, 6a ed., Atlas, 2017.

GRECH, V. Secular trends in newborn sex ratios. Early Human Development, v.90, n.11, p.755-760, 2014.

GRECH, V. Population stress, civil unrest and the male to female ratio at birth in Chile, Argentina, Australia and Finland. International Journal of Tropical Disease \& Health, v.6, n.1, p.27-34, 2015.

HAWLEY, A. Population composition. In: HAUSER, P.M.; DUDLEY DUNCAN, O. (Eds.), The study of population: an inventory and appraisal, University of Chicago Press, Chicago, pp. 361-382, 1959.

INSTITUTO BRASILEIRO DE GEOGRAFIA E ESTATÍSTICA (IBGE). Plataforma SIDRA. Estatísticas do Registro Civil. Disponível em: https://sidra.ibge.gov.br/tabela/2679 [24/10/2019a].

INSTITUTO BRASILEIRO DE GEOGRAFIA E ESTATÍSTICA (IBGE). Plataforma SIDRA. Censo demográfico. Disponível em: https://sidra.ibge.gov.br/tabela/200 [24/10/2019b].

LAZZAROTTI FILHO, A.; MASCARENHAS, F.; STIGGER, M.P.; SILVEIRA, R. DA; SILVA, A.M. Tendências no campo da educação física brasileira. Análise dos 
DOI: $10.12957 / \mathrm{e}-\mathrm{mosaicos} .2020 .45084$

documentos produzidos pela área 21 da Capes. Revista Brasileira de Ciências do Esporte, v.40, n.3, 233-241, 2018.

LOPES, O.U. Pesquisa básica versus pesquisa aplicada. Estudos Avançados, v.5, n.13, 219-221, 1991.

LOUZADA, M.L.C.; MARTINS, A.P.B.; CANELLA, D.S.; BARALDI, L.G.; LEVY, R.B.; CLARO, R.M.; MOUBARAC, J.-C.; CANNON, G.; MONTEIRO, C.A. Alimentos ultraprocessados e perfil nutricional da dieta no Brasil. Revista de Saúde Pública, v.49, 38, 2015. DOI:10.1590/S0034-8910.2015049006132

MASSARANI, L.; ARARIPE, C. Aumentar o diálogo com a sociedade é uma questão de sobrevivência para a ciência brasileira. Cadernos de Saúde Pública, v.35, n.6, e00089619, 2019.

MELO, A.C.M.; SILVA, G.D.M. DA; GARCIA, L.P. Mortalidade de homens jovens por agressões no Brasil, 2010-2014: Um estudo ecológico. Cadernos de Saúde Pública, v.33, n.11, p. :e00168316, 2017. DOI: https://doi.org/10.1590/0102-311X00168316

NÓVOA, A. (org.) Os professores e sua formação. Lisboa: DOM Quixote, 1992.

ORGANIZAÇÃO DAS NAÇÕES UNIDAS (ONU)-BRASIL. 17 objetivos para transformar nosso mundo. Disponível: https://nacoesunidas.org/pos2015/ [29/08/2019].

PENIN, S. A aula: espaço de conhecimento, lugar de cultura. São Paulo: Papirus, 1994.

PEREIRA, B.L.; SANTOS, A.C.D.; AQUINO, J.M.; ANDRADE NETO, G.R. DE; SOUZA, A.A.D. DE; RIOS, B.R.M.; LEÃO, C.D.A.; PEREIRA, S.G.S.; SIQUEIRA, L. DAS G; Piris, A.P. Colonização por microrganismos em colaboradores de saúde. Enfermagem Brasil, v.18, n.2, 213-219, 2019.

PEREIRA, J.E.D., ZEICHNER, K.M. (Ed.). A pesquisa na formação e no trabalho docente. Belo Horizonte: Ed. Autêntica, 2002.

PERRENOUD, P. Práticas pedagógicas, profissão docente e formação: perspectivas sociológicas. Lisboa: Ed. Dom Quixote, 1993.

PIANA, M.C. A pesquisa de campo. In: Piana, M.C. A construção do perfil do assistente social no cenário educacional. São Paulo, Ed Unesp e Cultura Acadêmica, 2009. Capítulo 5, p.167-210.

PIRES, V.B.; PRADO, G.M. Percepções de professores das séries iniciais sobre o ensino de ciências em uma escola de São Mateus - ES. Kiri Kerê Pesquisa e Ensino, n.4, 11-24, 2018. 
DOI: $10.12957 / \mathrm{e}-\mathrm{mosaicos} .2020 .45084$

REZENDE, C. DE P.; FREITAS, P.E.; VIÚDES, M. DE A.A.; PAULO, L.D.R. DE; COSTA, J.M. DA. Identificação de prescrições de medicamentos com risco de queda antes e após a internação hospitalar de idosos. Revista Brasileira de Farmácia, 100(1):30763090, 2019.

SAVIANI, D. Educação: do senso comum à consciência filosófica. São Paulo: Cortez, 1980.

SILVA, M.C. DA; CHACON, M.J.M.; PEDERNEIRAS, M.M.M.; LOPES, J.E. DE G. Procedimentos metodológicos para a elaboração de projetos de pesquisa relacionados a dissertação de mestrado em ciências contábeis. Revista de Contabilidade e Finanças, n.36, 97-104, 2004.

SPANHOL, R. DE C., FERRARI, C.K.B. Obesity and lifestyle risk factors among an adult population in Legal Amazon, Mato Grosso, Brazil. Revista de Salud Pública, 18:26-36, 2016.

VASCONCELLOS-SILVA, P.R.; CASTIEL, L.D.; GRIEP, R.H. A sociedade de risco midiatizada, o movimento antivacinação e o risco de autismo. Ciência \& Saúde Coletiva, v.20, n.2, 607-616, 2015.

Recebido em 28 de setembro de 2019 Aceito em 30 de outubro de 2019

Os direitos autorais de todos os trabalhos publicados na revista pertencem ao(s) seu(s) autor(es) e coautor(es), com o direito de primeira publicação cedido à e-Mosaicos.

Os artigos publicados são de acesso público, de uso gratuito, com atribuição de autoria obrigatória, para aplicações de finalidade educacional e não-comercial, de acordo com o modelo de licenciamento Creative Commons adotado pela revista. 